\title{
Satisfaction and Shortfall of OB-GYN Physicians and Radiologists
}

\author{
Christiane Herber-Valdez ${ }^{1}$, Sanja Kupesic-Plavsic ${ }^{2}$
}

\begin{abstract}
Ultrasound is pivotal to the practice of obstetrics-gynecology (OB-GYN). In the US, physicians who perform ultrasound are facing the unprecedented need for their services and skills. OB-GYNs, in particular, have been challenged to meet rising demands in women's healthcare, as a result of an increasing female population, while the supply of physicians is not keeping pace. The ACOG projects current shortages will continue to grow to a deficit of up to 22,000 OB-GYNs by 2050. The future of the OB-GYN workforce is compromised by an aging OB-GYN population, insufficient OB-GYN residency positions, and a decreasing number of young physicians choosing to specialize in OB-GYN.

As a consequence of mismatched supply and demand, practicing OB-GYNs are experiencing alarming rates of medical burnout, jeopardizing their mental health and wellness. The COVID-19 pandemic has further exacerbated risks to mental health; however, OB-GYNs have reported higher levels of burnout compared to other specialties, both before and after the pandemic. This article examines the threats to OB-GYN's central role in the provision of OB-GYN ultrasound, including factors contributing to insufficient growth of the OB-GYN workforce, and the resulting impacts on practicing OB-GYNs'job satisfaction and overall well-being.

Data on medical burnout affecting OB-GYNs, both before and after the COVID-19 pandemic, is presented along with a comparison of job satisfaction and wellness data collected from OB-GYNs and radiologists-the two groups of specialists performing female pelvic and OB ultrasound exams in the US.

Understanding the factors that discourage medical graduates from entering into OB-GYN residencies is critical, not only for recruitment but for the development of strategies to support currently practicing OB-GYNs. Prevention of medical burn-out among OB-GYNs will be essential to keep OB-GYNs practicing through retirement age while attracting others to the specialty. The creation of rewarding work environments, which allow for a healthy work-life balance, will be essential to meeting the demand for specialized women's health and reproductive care. Protecting the well-being of those practicing now, will be instrumental to the development of a sufficient OB-GYN workforce, and ensure its central role in the provision of OB-GYN ultrasound.

Keywords: Anxiety, Mental distress, Obstetrics-Gynaecology, Physicians, Radiologists, Shortfall, Ultrasound.

Donald School Journal of Ultrasound in Obstetrics and Gynecology (2021): 10.5005/jp-journals-10009-1822
\end{abstract}

\section{INTRODUCTION}

Ultrasound is pivotal to the practice of obstetrics-gynecology (OB-GYN). While the impact of ultrasound on women's imaging and the field of OB-GYN cannot be overstated, it is important to recognize the need for skilled ultrasound operators and interpreters, and the increasing demands on the healthcare professionals who conduct these procedures.

In the US, OB-GYN ultrasound is interpreted by two groups of medical professionals: OB-GYNs and radiologists. In an era of a growing, national physician shortage, medical providers are facing an unprecedented need for their services and skills, with OB-GYNs being particularly challenged to meet rising demands in women's healthcare. While the female population in the US continues to increase, over half of US counties do not have a single OB-GYN to provide healthcare for the over 10 million women who live in these counties. The ACOG projects current shortages will grow to a deficit of up to 22,000 OB-GYNs by $20500^{1,2}$

Factors that impact the shortfall in the OB-GYN workforce include an aging OB-GYN population, a diminishing pipeline of young physicians choosing the specialty, and a lack of OB-GYN residency slots, to name a few. ${ }^{1}$ Meanwhile, ever-increasing workloads are beginning to take their toll, with physicians experiencing burnout rates that are twice the rate of other working adults. ${ }^{3}$
${ }^{1}$ Department of Medical Education, Institutional Research, Effectiveness and Accreditation, Texas Tech University Health Sciences Center El Paso, 5001 El Paso Drive, El Paso, Texas, USA

${ }^{2}$ Department of Obstetrics and Gynecology, Paul L. Foster School of Medicine, Texas Tech University Health Sciences University El Paso, 5001 El Paso Drive, El Paso, Texas, USA

Corresponding Author: Christiane Herber-Valdez, Texas Tech University Health Sciences Center El Paso, Medical Center of the Americas Building, Room 254, El Paso, Texas, USA, Phone: +1-915-215-4796, e-mail: Christiane.Herber-Valdez@ttuhsc.edu

How to cite this article: Herber-Valdez C, Kupesic-Plavsic S. Satisfaction and Shortfall of OB-GYN Physicians and Radiologists. Donald School J Ultrasound Obstet Gynecol 2021;15(4):387-392.

Source of support: Nil

Conflict of interest: None

Even before the COVID-19 pandemic, close to half of OB-GYNs reported feeling burned out or depressed, citing the growing number of administrative tasks, lack of respect, and insufficient compensation/reimbursement as the main contributors. Alarmingly, half of those reporting burnouts considered it as having a strong or severe impact on their lives. ${ }^{4}$

The COVID-19 pandemic further exacerbated physician burnout and mental health risks. Especially those medical professionals

\footnotetext{
(c) The Author(s). 2021 Open Access This article is distributed under the terms of the Creative Commons Attribution 4.0 International License (https://creativecommons. org/licenses/by-nc/4.0/), which permits unrestricted use, distribution, and non-commercial reproduction in any medium, provided you give appropriate credit to the original author(s) and the source, provide a link to the Creative Commons license, and indicate if changes were made. The Creative Commons Public Domain Dedication waiver (http://creativecommons.org/publicdomain/zero/1.0/) applies to the data made available in this article, unless otherwise stated.
} 
working the front lines of the pandemic, are more likely to have depression, anxiety, and mental distress. Psychological distress from witnessing COVID-19-related deaths, long hours, and work-life imbalance have severely compromised mental health and well-being. ${ }^{5}$ Interestingly, both before and after the pandemic, OB-GYNs have reported higher levels of burnout compared to other specialties, including radiologists. ${ }^{4}$

In this article, we examine the challenges to OB-GYNs performing ultrasound in the US, including factors causing insufficient growth of a much needed OB-GYN workforce, as well as impacts on the job satisfaction and overall well-being of today's practicing OB-GYNs.

We include current data on medical burnout affecting OB-GYNs, both before and after the COVID-19 pandemic. We further provide an assessment of physician satisfaction and overall happiness, while comparing the two groups of specialists predominantly performing female pelvic and OB ultrasound exams in the US-OB-GYNs, and radiologists.

Our article concludes with recommendations for the prevention of burnout, to ensure ongoing, high-quality women's imaging and women's healthcare, as well as the future growth of an OB-GYN workforce.

\section{Who is Performing Ob-Gyn Ultrasound?}

Around the globe, OB-GYN ultrasound is performed and interpreted by various providers. Including OB-GYNs, radiologists, emergency and family medicine physicians, sonographers, midwives, and nurses (Fig 1). ${ }^{6}$ In the United States (US) and Canada, OB-GYN ultrasound is typically performed by trained sonographers and interpreted by OB-GYNs and radiologists. OB-GYNs performing ultrasound claim that ultrasound is an adjunct to bimanual pelvic examinations, and that their training, familiarity with the anatomy of the female pelvis, and clinical experience enable them to better correlate ultrasound findings with patients' clinical signs and symptoms.

Given the importance of appropriate performance and interpretation of ultrasound results, regulatory bodies across nations have developed standards for ultrasound examinations. In the US, the American College of Obstetricians and Gynecologists (ACOG) and the American Institute of Ultrasound in Medicine (AIUM) have developed comprehensive professional competencies for OB-GYN ultrasound scanning..$^{7-9}$ In European and Asian countries regulatory bodies collaborate with international societies, such as the International Society for Ultrasound in Obstetrics and Gynecology (ISUOG) to develop comprehensive training requirements. ${ }^{10}$ Australia and New Zealand are unique in offering a formally recognized fellowship in ultrasound, entitled Certification in Obstetrical and Gynaecological Ultrasound, for OB-GYNs intending to integrate ultrasound into their day-to-day practice. ${ }^{11}$

In addition, radiology associations in Europe (European Society of Radiology, ESR), and the US (American College of Radiology, ACR) specify standards for education and training, determine minimum requirements for ultrasound equipment, the impact of new tools in daily practice, practical aspects for the performance of ultrasound examinations (including image recording and storage, reporting, etc.), equipment maintenance (including cleaning and disinfection), and medico-legal issues. ${ }^{12-14}$

While modern obstetrics and gynecology practice is considered "virtually impossible without the use of ultrasound", ${ }^{10}$ it has also been utilized in other specialties. In many countries, including the US, women's healthcare is an integral part of family medicine physicians' scope of practice-especially in underserved and rural communities. ${ }^{15,16}$ The advantages of this approach are improved accessibility and continuity of women's healthcare, immediate assessment of urgent clinical presentations, and cost reduction. ${ }^{15}$

Other specialties include emergency medicine physicians, who commonly utilize Point-of-Care Ultrasound (PoCUS) at the bedside to evaluate patients presenting acute pelvic pain, abnormal bleeding, or early pregnancy complications. This approach has led to improved patient outcomes. For example, among patients with acute pelvic pain and vaginal bleeding, who require ultrasound as a part of their diagnostic evaluation in emergency medicine, PoCUS has been shown to result in a statistically significant decrease in overall length of hospital stay, and reduced need for radiology ultrasound in nearly one-third of patients. ${ }^{17}$ While it is important that staff performing the scans are well-trained and knowledgeable about the indications for PoCUS and formal imaging, ${ }^{18}$ PoCUS is particularly useful in underserved areas because it is relatively cheap and accessible.

Unfortunately, a growing shortage of physicians in the US is presenting significant challenges to meeting today's healthcare demands, including in the areas of women's health and OB-GYN. In fact, given a critical shortage of OB-GYNs, the implementation of OB-GYN ultrasound in low-income countries and rural areas has also been facilitated by midwives and nurses. ${ }^{19}$

\section{Growing Physician Shortage in the US}

Physician availability has been recognized as one of the most common nonfinancial issues leading to unmet or delayed healthcare among US adults. ${ }^{20}$ A recent report from the Association of American Medical Colleges (AAMC) confirms the US could face an estimated shortage of 54,100-139,000 physicians by $2033 .^{21}$ This projected shortfall includes an estimated shortage of 21,400-55,200 primary care physicians, and a shortage of $33,700-86,700$ nonprimary care specialty physicians. The AAMC cites population growth and aging as the primary drivers of increasing demand for healthcare in coming years. By 2033, changing demographics alone are estimated to increase the national demand for physicians by $17 \%$, with a projected $19 \%$ growth in demand for primary care physicians, and a projected $25 \%$ growth in demand for medical specialists.

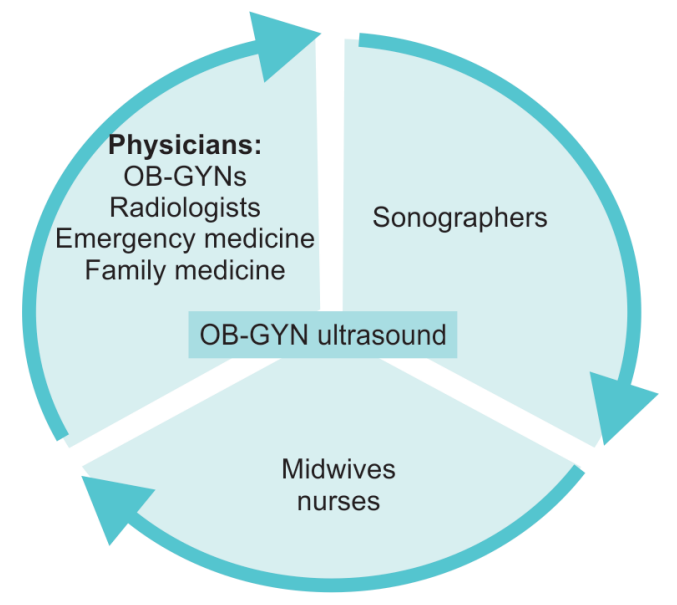

Fig. 1: Obstetrics-gynecology (OB-GYN) ultrasound is performed and interpreted by physicians (OB-GYNs, radiologists, emergency medicine and family medicine), sonographers, midwives and nurses 
Meanwhile, the supply of physicians will in large part depend on the retirement intentions of older physicians. Currently, a substantial portion of the physician workforce is nearing retirement age, with more than two out of every five active physicians turning 65 years or older within the next decade. Given the potential impact of these factors, amidst national goals focused on achieving health equity through increased access to care, the agency predicts physician demand will continue to grow faster than supply, "despite the dizzying pace of debate around the organization, regulation, finance, and technology of healthcare delivery". ${ }^{21}$

\section{Physician Shortage: Impact on the OB-GyN WorkForce}

The increasing shortage of physicians is having negative impacts across specialties, including in OB-GYN. In 2017, the American College of Obstetricians and Gynecologists (ACOG) reported that half of US counties lack a single OB-GYN. ${ }^{1,22}$ These counties are home to more than 10 million women, of whom many may need OB-GYNs for primary care. ${ }^{1}$ Moreover, the ACOG projects current shortages will grow to a deficit of up to 22,000 OB-GYNs by $20500^{1,2}$

Not only is the shortage a national problem, but looking at the impact of the OB-GYN deficit on large urban areas, researchers found none of the 50 US metropolitan areas included in the study to have at least $30 \%$ of their OB-GYN workforce below the age of $40 .{ }^{23}$ However, it is women in rural areas that are hit hardest, with over half living more than 30 minutes from a hospital with perinatal services. ${ }^{24}$

Meanwhile, the ACOG projects a $6 \%$ estimated increase in demand for women's healthcare in the US over the next 10 years, due to ongoing growth in the female population. ${ }^{25}$

The mismatch of projected supply and demand is particularly alarming, as it will have significant implications for women's imaging and women's healthcare in general. In the words of AAMC Chief Health Care Officer Janis Orlowski, MD "The growing OB-GYN shortage represents a serious threat to women, many of whom are low-income and in remote rural areas, and who need quality prenatal care, cancer screening, and other vital services".

\section{Understanding the Shortage}

Several factors continue to impact the growing OB-GYN shortage. Many OB-GYNs are nearing retirement age. ${ }^{1,26}$ In 2018, the AAMC reported an average age of 51, and the group's tendency to retire beginning at age 59.' A 2019 workforce study, analyzing the factors impacting the OB-GYN specialty, found $35 \%$ of OB-GYNs are 55 years and older. This would not be concerning if younger OB-GYNs were available to fill these soon-to-be vacancies; however, less than one in five (19\%) of OB-GYNs are younger than 40 years old. ${ }^{1}$

In addition to an aging OB-GYN workforce, too few residency slots are available to fill the pipeline. While the number of US females over age 18 increased by 33 million, OB-GYN first-year residency positions grew by less than 200 between 1992 and 2016. Compounding the problem is an increasing number of OB-GYN residents pursuing subspecialty training - 7\% in 2000 vs $20 \%$ in 2012-in large part because OB-GYNs are among the least compensated among surgical specialties. As a result, increasing numbers of OB-GYNs are completing fellowship training in better-compensated subspecialties. ${ }^{1}$

Others may not choose OB-GYN specialization, or OB-GYNs might leave their practice, due to the specialty's high likelihood of being sued. Before they turn 55, over half of OB-GYNs have already been sued, and nearly two out of three OB-GYNs face legal action at some point, the highest rate of all specialties. ${ }^{27}$

Work-life balance and professional burnout continue to be key challenges for OB-GYNs. According to the ACOG, $40-70 \%$ of OB-GYNs will experience some form of burnout, and female OB-GYNs are more likely to reduce their clinical work hours or leave their practice due to lack of work-life balance and family responsibilities. ${ }^{25}$ Interestingly, about $59 \%$ of practicing OB-GYNs are women, a much higher proportion compared to the percentage of women practicing general surgery (22\%) and radiology (26.5\%). Given this percentage, and considering the number of females enrolled in OB-GYN residencies, the ACOG predicts $66 \%$ of OB-GYNs will be female by $2027 .^{25}$

It is, in fact, well-documented that surgical-related residents and specialists (General Surgery and OB-GYN) demonstrate higher rates of job dissatisfaction and burnout compared to nonsurgical specialties. ${ }^{28}$ In addition, $72 \%$ of OB-GYNs have reported some degree of anxiety about their future. ${ }^{28}$

\section{Medical Burnout during the COVID-19 PANDEMIC}

In 2017, the American College of Obstetricians and Gynecologists (ACOG) stated:
"The pace of life and its stresses, impact from multitasking, overwhelming information exposure, and electronic medical record expectations have led to some degree of physical or emotional exhaustion or lack of motivation. Physicians have burnout rates that are twice the rate of other working adults, and no area of medicine is immune. It is estimated that $40-75 \%$ of OB-GYNs experience some form of professional burnout (For example, losing control, conflicting demands on time, or diminishing sense of worth)." ${ }^{3}$

The COVID-19 pandemic propelled burnout among medical professionals to unprecedented heights. A recent survey of 1,327 US healthcare care professionals on the front lines during the COVID-19 pandemic revealed that medical burnout-defined as mental and physical exhaustion from chronic workplace stress has reached epidemic proportions. ${ }^{29}$ According to survey results, an overwhelming $55 \%$ of front-line healthcare workers reported feeling burned out, and about half reported feeling anxious (49\%). Those aged 18-29 reported the highest burnout rate (69\%), and the highest negative impact on mental health (75\%) Overall, the majority of participants (62\%) reported some mental health repercussions.

Medical professionals providing direct care for COVID-19 patients are more likely to have depression, anxiety, and mental distress. Experiencing psychological distress from witnessing COVID-19-related deaths, working long hours, and work-life imbalance have compromised mental health and well-being. In addition, despite increased workloads, many have suffered financial distress, including salary cuts and furloughs. The child care crises placed particular strains on women in medicine. ${ }^{5}$

According to Kaushik, "health care workers are an indispensable part of the nation's workforce and have saved countless lives, before the pandemic and during it. Understanding medical burnout and having strategies at the organizational level to prevent it will help avoid future catastrophes". 


\section{Comparison of Burnout, Personal Well-being, and Job Satisfaction among Ob-Gyns and Radiologists: Before and DURING The COVID-19 PANDEMIC}

To explore how physicians are coping with burnout, maintaining personal wellness, and viewing their workplace and their future amid the COVID-19 pandemic, Medscape surveyed more than 12,000 physicians in over 29 specialties. ${ }^{4}$

When physicians were asked about their overall happiness outside of work before the pandemic, $81 \%$ of OB-GYNs and $82 \%$ of radiologists said they were somewhat or very happy. ${ }^{30}$ In comparison, endocrinologists ranked highest (89\%), and infectious disease specialists ranked lowest (69\%) (Fig. 2).

Reported levels of happiness dropped during the pandemic, with $57 \%$ of OB-GYNs and $60 \%$ of radiologists indicating they were happy or somehow happy. ${ }^{4}$ Endocrinologists continued to rank highest, with $73 \%$ reported being very or somewhat happy, while infectious disease specialists (45\%), pulmonologists (47\%), and intensivists ranked lowest.

Forty-four percent of OB-GYNs said they were burned out or burned out and depressed, compared to only $37 \%$ of radiologists who reported burnout and/or depression ${ }^{4}$ (Fig. 3).

Among OB-GYNs, more females (57\%) expressed burnout than their male peers (43\%). ${ }^{4}$ Moreover, $72 \%$ of OB-GYNs considered their burnout serious enough to have at least a moderate impact on their lives, with $52 \%$ considering it as having a strong/severe impact. ${ }^{4}$

The large majority of OB-GYNs (82\%) reported being burned out before the beginning of the pandemic. ${ }^{30}$ When OB-GYNs were asked what contributed to their burnout, they pointed out the growing number of administrative tasks, lack of respect, and insufficient compensation/reimbursement. ${ }^{30}$ For radiologists, spending too many hours at work, lack of respect from the colleagues at the workplace, and lack of autonomy were the major contributors to burnout. ${ }^{30}$

It is worrisome that about $20 \%$ of burned-out and/or depressed OB-GYNs reported suicidal thoughts. ${ }^{30}$ This number is significantly higher compared to radiologists reporting burnout, depression, or both, of which $12 \%$ reported suicidal thoughts. About $1 \%$

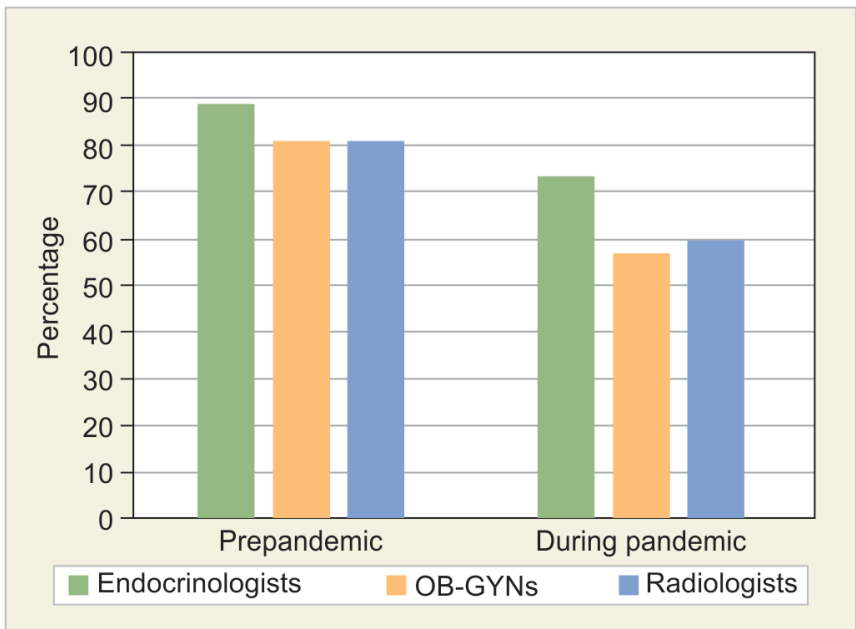

Fig. 2: Overall happiness outside of work before the pandemic for endocrinologists, obstetricians and gynecologists and radiologists (adapted from Refs. Martin and Koval ${ }^{4}$, and Peckam ${ }^{30}$ ) (the same percentage of burned-out and/or depressed OB-GYNs and radiologists) reported having attempted suicide.

When asked how they cope with the symptoms of burnout, $48 \%$ of OB-GYNs and $42 \%$ of radiologists reported isolating themselves from others. ${ }^{30}$ Forty-three percent of OB-GYNs and 50\% of radiologists exercise, while $42 \%$ and $38 \%$, respectively, talk with family members and close friends. About $21 \%$ of OB-GYNs are currently seeking or plan to seek professional help, compared with $11 \%$ of radiologists. Fifty-six percent of OB-GYNs and $72 \%$ of radiologists have not sought professional care in the past, because they did not consider their symptoms severe enough or felt too busy. Significantly more radiologists (56\%) take five or more weeks of vacation per year, compared with OB-GYNs (18\% ${ }^{4}$ (Fig. 4).

About $20 \%$ of OB-GYNs and radiologists consume five or more drinks per week. This percentage is about the same as the percentage of respondents who reported not consuming alcohol at all.

The great majority of OB-GYNs and radiologists ( $87 \%$ and $88 \%$, respectively) report being in a committed relationship. Eighty-three percent of OB-GYNs and $90 \%$ of radiologists described their relationships as very good or good. Although, a notable percentage

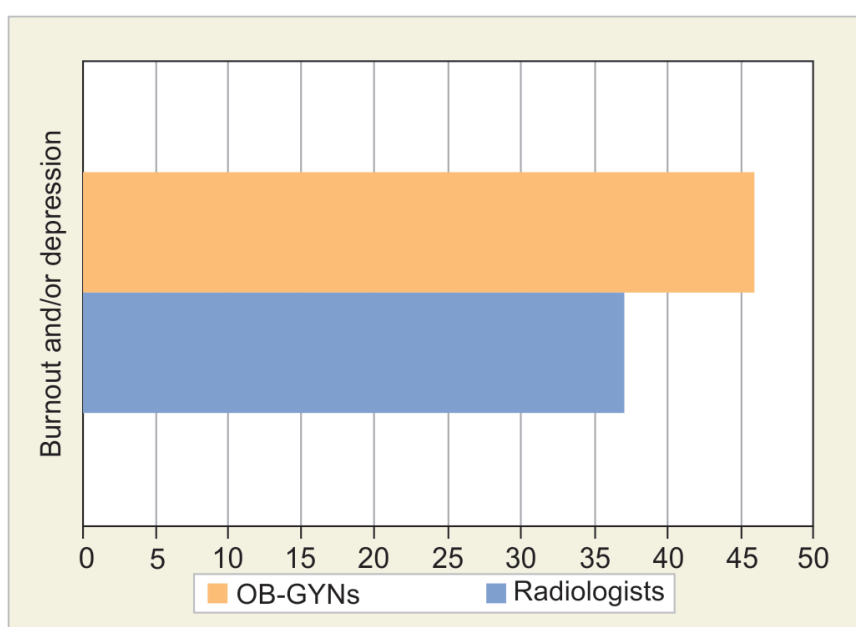

Fig. 3: Burnout and/or depression reported by OB-GYNs and radiologists during COVID-19 pandemic (adapted from Ref. Martin and Koval ${ }^{4}$ )

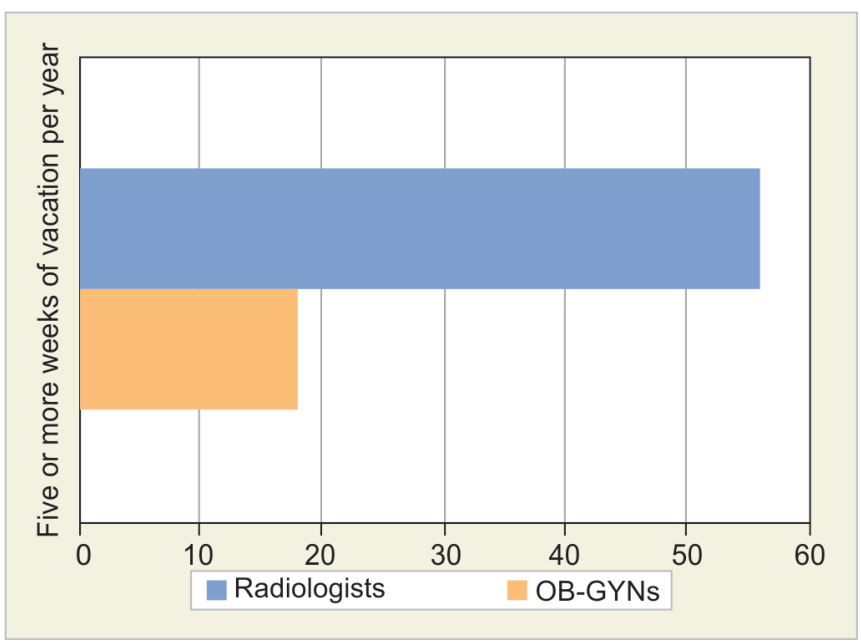

Fig. 4: Prolonged vacation (five or more weeks per year) by OB-GYNs and radiologists (adapted from Ref. Martin and Koval ${ }^{4}$ ) 
of OB-GYN and radiologists' partners/spouses (about 40\%) also work in health care. This may have additional, potentially negative impacts on work-life balance, family resilience, and well-being during a pandemic., ${ }^{40}$

Among both OB-GYNs and radiologists (46\%), work-life balance was considered their top concern. Forty-eight percent of both OB-GYNs and radiologists reported they would take a salary reduction to improve their work-life balance. ${ }^{30}$

The pandemic further amplified disparities among men and women physicians. With a need for daytime at-home childcare, work-life balance was particularly impacted. Many women continue to bear a disproportionate share of family responsibilities, which may have important repercussions for patient care, and work-life balance among female physicians. ${ }^{31}$

\section{Burnout Prevention}

Risk factors for burnout among both OB-GYNs and radiology specialists are associated with work overload, long hours, night calls, lack of control, and prolonged stress. ${ }^{4,30}$ Other risk factors include the introduction of new technologies (e.g., 3/4-D ultrasound), requiring additional training, and the acquisition of new skills. A good orientation to a new technique, availability of adequate training, and mentoring decrease stress and improve resilience. ${ }^{32}$

Reducing isolation and improving interaction with sonographers and referring physicians increase the satisfaction of the OB-GYNs or radiologists interpreting ultrasound. Intellectual stimulation and opportunity for career development are also associated with increased job satisfaction. ${ }^{32}$

Positive feedback and demonstration of appreciation for accurate and timely preoperative diagnosis increase job satisfaction, and decrease turnover. Recognizing the accomplishments of the individual faculty and providing credit for excellent service and good work are shown to improve morale and commitment to the department, organization, and/or practice.

Additional strategies for burnout prevention include improving the efficacy of the service through the introduction of high-performance dictation, voice recognition, and PACS medical image archiving systems, using physician and radiology assistants, scribes, nurse practitioners, and other administrative personnel to decrease the amount of administrative work of physicians performing women's imaging.

\section{Conclusion}

OB-GYN ultrasound plays a central role in the management of women's health. While ultrasound is performed by a variety of providers in other countries, in the US, the two groups of physicians who interpret OB-GYN ultrasound are OB-GYNs and radiologists. Although, for OB-GYNs, ultrasound is not only an indispensable tool but an essential part of the practice. For OB-GYNs, ultrasound is integral to the medical and surgical care of female reproductive health.

With the nation's female population rising, demands on OB-GYNs have also increased. Yet, amidst a national critical shortage of physicians, the number of OB-GYNs is not keeping pace. With an aging OB-GYN workforce and a lack of a "pipeline" of residents to fill upcoming OB-GYN vacancies, the profession is facing critical shortfalls, causing serious concerns for the future of women's imaging and women's healthcare in general.

Further compounding a bleak forecast, are data showing the effects of the mismatch between OB-GYN demand and provider supply, with OB-GYNs reporting higher levels of job dissatisfaction, stress, and mental health deficits, when compared to other specialty providers. Even before the onset of the COVID-19 pandemic, an overwhelming majority of OB-GYNs reported suffering from medical burnout.

The COVID-19 pandemic has further challenged an already burdened OB-GYN workforce. In addition to the detrimental mental health effects on all healthcare professionals during the pandemic, providers performing OB-GYN ultrasound were considered a highly vulnerable frontline group with a high risk of exposure to COVID-19, leading to an increased risk of burnout and poor emotional state. ${ }^{33}$

As we emerge from the COVID-19 crisis, we are aware of the strain and potentially long-term negative impacts on healthcare professionals' well-being. However, for OB-GYNs in particular, these impacts have further compounded pre-existing issues. We need to be cognizant of the circumstances that have been and will continue to impact job satisfaction.

Understanding the factors that discourage medical graduates from entering into OB-GYN residencies is important, not only to recruit and develop future generations of OB-GYNs but also to better support those currently practicing. Prevention of medical burn-out among OB-GYNs will be essential to keep OB-GYNs practicing through retirement age, while also attracting others to the specialty. Work environments and cultures where OB-GYNs feel valued, respected, and which allow for a healthy balance of professional and personal lives, will be crucial. Especially, in our roles as instructors, supervisors, managers, and administrators, we have to commit to creating professional, safe, and healthy spaces, which both attract and protect.

In sum, given the current state, and the need to grow an OB-GYN workforce equipped to meet tomorrow's demand for specialized women's health and reproductive care, it is essential that we take actions to ensure the well-being of those practicing now while securing the future of the OB-GYN profession and its central role in the provision of OB-GYN ultrasound.

\section{References}

1. Marsa, L. Labor pains: The OB-GYN shortage. Aamc.org 2018. Available from: https://www.aamc.org/news-insights/labor-painsob-gyn-shortage [cited 15.06.21].

2. Rosenberg J. Physician shortage likely to impact OB/GYN workforce in coming years. Am J Manag Care 2019. Available from:https:// www.ajmc.com/view/physician-shortage-likely-to-impact-obgynworkforce-in-coming-years [cited 15.06.21].

3. Why OB-GYNs are Burning Out.Acog.org 2019. Available from: https://www.acog.org/news/news-articles/2019/10/why-ob-gynsare-burning-out [cited 15.06.21].

4. Martin KL, Koval ML. Obstetrician \& gynecologist lifestyle, happiness \& burnout report 2021. Medscape.com. Available from: https:// www.medscape.com/slideshow/2021-lifestyle-obgyn-6013515 [cited 10.05.21]

5. Kaushik D. Medical burnout: breaking bad. Aamc.org. Available from: https://www.aamc.org/news-insights/medical-burnout-breakingbad [cited 10.05.21].

6. Leonardi M, Murji A, D'Souza R. Ultrasound curricula in obstetrics and gynecology training programs: obstetrics and gynecology ultrasound training. Ultrasound Obstet Gynecol 2017. Available from: Available from: DOI: 10.1002/uog.18978

7. Educational Objectives: Core Curriculum in Obstetrics and Gynecology. 10th ed. Washington, DC, USA: American College of Obstetricians and Gynecologists 2013. 
8. AIUM-ACR-ACOG-SMFM-SRU Practice parameter for the performance of standard diagnostic obstetric ultrasound examinations. J Ultrasound Med 2018;37(11):E13-E24. DOI: 10.1002/jum.14831

9. Practice parameter for the performance of ultrasound of the female pelvis. Aium.org 2013:301-498. Available from: http://www.aium.org [cited 18.05.21].

10. Salvesen KA, Lees $C$, Tutschek B. Basic European ultrasound training in obstetrics and gynecology: where are we and where do we go from here? 2010:525-529.

11. The Royal Australian and New Zealand College of Obstetricians and Gynaecologists. Certification in Obstetrical and Gynaecological Ultrasound Training Program 2017. Edu.au 2017. Available from: https://www.ranzcog.edu.au/Training/Subspecialist-Training/ Curriculum-Handbooks [cited 18.05.21].

12. European Society of Radiology (ESR). Position statement and best practice recommendations on the imaging use of ultrasound from the European Society of Radiology ultrasound subcommittee. Insights Imaging 2020;11(1):115. DOI: 10.1186/s13244-020-00919-x

13. Practice guideline for the performance of the standard diagnostic obstetrical ultrasound 2018.Pedrad.org. Available from: https:// www.pedrad.org/Portals/5/Subspecialties/OB\%20Ultrasound\%20 practice\%20guidelines.pdf [cited 18.05.2021].

14. Practice guideline for the performance of ultrasound of the female pelvis. Acr.org 2019. Available from: https://www.acr.org/-/media/ ACR/Files/Practice-Parameters/US-Pelvis.pdf [cited 18.05.21].

15. Obstetric ultrasound examination (Position Paper).Aafp.org. Available from: https://www.aafp.org/about/policies/all/obstetric-ultrasound. html [cited 18.05.21].

16. Tong STC, Makaroff LA, Xierali IM, et al. Proportion of family physicians providing maternity care continues to decline. J Am Board Fam Med 2012;25(3):270-271. DOI: 10.3122/jabfm.2012.03.110256

17. Wilson SP, Connolly K, Lahham S, et al. Point-of-care ultrasound versus radiology department pelvic ultrasound on emergency department length of stay. World J Emerg Med 2016;7(3):178-182. DOI: 10.5847/wjem.j.1920-8642.2016.03.003

18. Collins K, Collins C, Kothari A. Point-of-care ultrasound in obstetrics. Australas J Ultrasound Med 2019;22(1):32-39. DOI: 10.1002/ajum. 12133

19. Holmlund S, Ntaganira J, Edvardsson K, et al. Health professionals' experiences and views on obstetric ultrasound in Rwanda: a cross-sectional study. PLoS One 2018;13(12):e0208387. DOI: $10.1371 /$ journal.pone.0208387

20. Kullgren JT, McLaughlin CG, Mitra N, et al. Nonfinancial barriers and access to care for US adults. Health Serv Res 2012;47(1pt2):462-485. DOI: 10.1111/j.1475-6773.2011.01308.x

21. The complexities of physician supply and demand: projections from 2018 to 2033. Association of American Medical Colleges 2020. Available from: https://www.aamc.org/media/45976/download.
22. Nation's OB-GYNs Meet in Washington to Bridge the Gap in Women's Health. Acog.org. Available from: https://www.acog.org/news/newsreleases/2015/03/nations-obgyns-meet-in-washington-to-bridgethe-gap-in-womens-health [cited 10.06.21].

23. New research finds growing U.S. physician shortage hits maternity care. Doximity.com. Available from: https://press.doximity.com/ articles/physician-shortage-hits-maternity-care [cited 18.05.21].

24. Phelan ST, Wetzel LM. Maternal death in rural America. Contemporaryobgyn.net. Available from: https://www. contemporaryobgyn.net/view/maternal-death-rural-america [cited 10.06.21].

25. Orvos JM. ACOG Releases new study on OB/GYN workforce. Contemporaryobgyn.net. Available from: https://www. contemporaryobgyn.net/view/acog-releases-new-study-obgynworkforce. [cited 18.05.21].

26. 2018 OB-GYN Workforce study: looming physician shortages: a growing women's health crisis. Amazonaws.com 201. Available from: https://s3.amazonaws.com/s3.doximity.com/press/OB-GYN_ Workload_and_Potential_Shortages_2018.pdf [cited 15.06.21].

27. Guardado JR. Policy research perspectives: medical liability claim frequency among U.S. physicians. Ama-assn.org. Available from: https://www.ama-assn.org/sites/ama-assn.org/files/corp/mediabrowser/public/government/advocacy/policy-research-perspectivemedical-liability-claim-frequency.pdf [cited 10.06.21].

28. Hutchinson-Colas J, Brug P, Patel NM. Job satisfaction amongst OB/ GYN and surgical residents at an academic center. Obstet Gynecol 2016;128(1):47S-47S. DOI: 10.1097/01.AOG.0000502689.53932.7e

29. Kirzinger A, Kearney A, Hamel L, et al. KFF/the Washington Post Frontline Health Care Workers Survey - toll of the pandemic.Kff. org 2021. Available from: https://www.kff.org/report-section/kff-thewashington-post-frontline-health-care-workers-survey-toll-of-thepandemic/ [cited 18.05.21].

30. Peckam C. Ob/Gyn Lifestyle Report 2016: bias and burnout. Medscape.com 2016. Available from: https://www.medscape.com/ features/slideshow/lifestyle/2016/womens-health\#page=4 [cited 18.05.21].

31. Lubell J. Women physicians and the pandemic: a snapshot. Mdedge. com 2021. Available from: https://www.mdedge.com/psychiatry/ article/234774/coronavirus-updates/women-physicians-andpandemic-snapshot?sso=true [cited 18.05.21].

32. Harolds JA, Parikh JR, Bluth El, et al. Burnout of radiologists: frequency, risk factors, and remedies: a report of the ACR commission on human resources. J Am Coll Radiol 2016;13(4):411-416. DOI: 10.1016/j. jacr.2015.11.003

33. Palsson O,Ballou S, GrayS.TheU.S.National Pandemic Emotional Impact Report. Pandemicimpactreport.com 2020. Available from: https:// www.pandemicimpactreport.com/report/PalssonBallouGray_2020_ PandemiclmpactReport.pdf [cited 18.05.21]. 